\title{
Oxytocin receptor gene polymorphism modulates the effects of social support on heart rate variability
}

Magdalena K. Kanthak ${ }^{\mathrm{a}, \mathrm{b}^{*}}$, Frances S. Chen ${ }^{\mathrm{a}, \mathrm{c}^{*}}$, Robert Kumsta ${ }^{\mathrm{d}}$, LaBarron K. Hille,f, Julian F.

Thayer $^{\mathrm{g}}$, and Markus Heinrichs ${ }^{\mathrm{a}, \mathrm{h}}$

${ }^{a}$ Department of Psychology, Laboratory for Biological and Personality Psychology, University of Freiburg, Germany

${ }^{b}$ Department of Biological Psychology, Technical University of Dresden, Germany

'Department of Psychology, University of British Columbia, Canada

${ }^{\mathrm{d} D e p a r t m e n t ~ o f ~ G e n e t i c ~ P s y c h o l o g y, ~ F a c u l t y ~ o f ~ P s y c h o l o g y, ~ R u h r-U n i v e r s i t y ~ B o c h u m, ~ G e r m a n y ~}$

${ }^{\mathrm{e}}$ Center for the Study of Aging and Human Development, Duke University Medical Center, Durham, North Carolina, USA

${ }^{\mathrm{f}}$ Department of Psychiatry and Behavioral Sciences, Duke University Medical Center, Durham, North Carolina, USA

'Department of Psychology, The Ohio State University, Columbus, USA

${ }^{h}$ Freiburg Brain Imaging Center, University Medical Center, University of Freiburg, Germany

*These authors contributed equally to the work.

Corresponding Authors:

Frances Chen

Department of Psychology

University of British Columbia

3521-2136 West Mall

Vancouver BC Canada V6T 1Z4

E-mail address: frances.chen@psych.ubc.ca

Phone: +1-604-822-2549

Markus Heinrichs

Laboratory for Biological and Personality Psychology

Department of Psychology

University of Freiburg

Stefan-Meier-Str. 8

D-79104 Freiburg, Germany

E-mail address: heinrichs@ psychologie.uni-freiburg.de

Phone: +49-761-203-3024 


\begin{abstract}
A large body of empirical research has demonstrated stress-buffering effects of social support.

However, recent studies suggest that genetic variation of the oxytocin system (specifically, a common single nucleotide polymorphism, rs53576, of the oxytocin receptor gene) modulates the efficacy of social support. The timing and neurobiological basis of this genetic modulation was investigated using a standardized, laboratory-based psychological stress procedure (Trier Social Stress Test for Groups, TSST-G). To index potential stress buffering effects of social support mediated by the oxytocin system, heart rate variability (HRV) was obtained before and during the TSST-G from 40 healthy participants. Results indicate that social support is associated with higher HRV only in G allele carriers. Specifically, social support increased heart rate variability solely during direct social interaction and only in individuals with at least one copy of the $G$ allele of rs53576. These findings confirm that the stress-attenuating effects of social support are modulated by genetic variation of the oxytocin system.
\end{abstract}

\title{
Keywords
}

Stress-buffering, molecular genetics, oxytocin, autonomic, heart rate variability, individual differences 


\section{Introduction}

The powerful influence of social support as a protective factor for maintaining and restoring health is well established (Cohen, 1988; Uchino et al., 1996; Weihs et al., 2005). Converging evidence from both laboratory-based and epidemiological research suggests that one mechanism through which social support exerts its influence on health is by attenuating physiological stress reactivity (Heinrichs et al., 2003; Kirschbaum et al., 1995; Lepore et al., 1993).

Although social interactions have stress-attenuating effects for most individuals, empirical research has revealed individual differences in these effects (Taylor et al., 2007; Ditzen et al., 2008; Kim et al., 2008; Chen et al., 2011). A growing body of research suggests that genetically-based differences in the oxytocin system may contribute to these individual differences. The fact that oxytocin exhibits pro-social and stress-attenuating effects (Kumsta and Heinrichs, 2013; Heinrichs et al., 2009; Meyer-Lindenberg et al., 2011), makes this neuropeptide a plausible mediator of the stressbuffering effects of social support. Additional support comes from research showing that in combination with social support, oxytocin attenuates autonomic stress responses (Heinrichs et al. 2003; Meyer-Lindenberg et al., 2011).

Research on genetic variations of the oxytocin receptor $(O X T R)$, through which oxytocin exerts a range of effects throughout the body and brain (Inoue et al., 1994; Gimpl and Fahrenholz, 2001), seems especially promising. One of the most-studied variations of OXTR is the single nucleotide polymorphism (SNP) rs53576 (G/A). Recent studies have linked this SNP to variability in both social behaviour (Bakermans-Kranenburg and van Ijzendoorn, 2008; Meyer-Lindenberg et al., 2011) and stress reactivity (Rodrigues et al., 2009; Norman et al., 2012; Kumsta \& Heinrichs, 2013). Specifically, the A allele has been associated with reduced positive affect (Lucht et al., 2009), reduced maternal sensitivity (Bakermans-Kranenburg and van IJzendoorn, 2008), and reduced empathic accuracy (Rodrigues et al., 2009). The A allele has also been associated with a higher risk for autism spectrum disorder (e.g., Wu et al., 2005) and depression (e.g., Saphire-Bernstein et al., 2011), both of which are associated with social functioning deficits.

Given that rs53576 has been shown to predict both stress buffering and prosocial behaviour, this SNP may also contribute to individual differences in the stress-buffering effects of social support. In fact, research from our lab showed that only individuals with at least one copy of the $\mathrm{G}$ allele of rs53576 displayed lower cortisol responses to stress following social support, relative to individuals of the same genotype receiving no social support (Chen et al., 2011). However, because cortisol reactions can be measured only with a substantial delay (in the range of 15-20 minutes) after the actual stressful event, the precise timing of the interaction between social support and rs53576 remains unclear.

Relevant to the search for the physiological locus of the aforementioned modulating effect of rs53576 is research in non-human mammals demonstrating a multilateral distribution of the oxytocin 
receptor in various brain regions that are associated with central nervous system (CNS) control of stress, including the amygdala, the paraventricular nucleus (PVN), nucleus tractus solitarious (NTS), the dorsal motor nucleus of the vagus (DMX), and the nucleus ambiggus (NA) (Luiten et al 1985; Higa et al 2002; Landgraf and Neumann, 2004; Coote, 2013). This is consistent with the possibility that variants of rs53576 could modulate the stress-buffering effects of social support at a central nervous system level.

Further support comes from research in non-human mammals showing that oxytocin modulates amygdala-mediated activation of the peripheral stress reaction (Viviani et al., 2011). However, none of the previous studies directly investigated the effects of the SNP rs53576 in combination with social support on CNS stress regulation. The use of heart rate variability (HRV) as an indicator of differences in stress regulation via recruitment of the parasympathetic system may help bridge this gap in knowledge.

Empirical evidence suggests that CNS stress regulation can be indexed by vagal functioning level (Thayer and Lane, 2009). Because the vagus nerve modulates the heart rate on a time scale of milliseconds, high frequency changes in heart rate, known as heart rate variability (HRV), are a valid index of parasympathetic activation level (Thayer et al., 2008) and therefore for CNS stress regulation (Friedman and Thayer, 1998; Gianaros et al., 2004), at a high temporal resolution. A large body of evidence associating positive social interactions with increased HRV (Kok \& Fredrickson, 2010; Smith et al., 2011; Maunderer et al., 2012) further illustrates the usefulness of this index in the present research design. The basic data used for calculating HRV is the sequence of time intervals between consecutive heart beats, also known as inter-beat intervals (for more information on HRV, see Task Force of the European Society of Cardiology and the North American Society of Pacing and Electrophysiology, 1996).

Furthermore, stimulation of oxytocin neurons has been shown to induce heart rate slowing (bradycardia) and increased vagal tone (Rogers and Herman, 1986; Higa et al., 2002). Importantly, it has been shown that these oxytocin neurons are associated with stress buffering in that they are more active during stressful events and thus serve to reduce the impact of such events by lowering heart rate via increased vagal tone (Higa et al., 2002), which strongly suggests the capability of HRV to index stress-buffering effects of social interactions mediated by oxytocin. The idea that oxytocin may play a role in modulating outflow of vagal efferent activity on a central nervous level was proposed more than a decade ago within the framework of Polyvagal Theory (Porges, 1997; Carter, 1998). Notably, however, no studies have yet examined the association between naturally-occurring individual differences in the functioning of the oxytocin system and measures of cardiac vagal outflow as indexed by HRV in the context of social stress buffering.

Therefore, the present study investigated via heart rate variability the CNS basis and the timing of the interaction of social support and rs53576 on stress reactivity. We predicted that 
individuals with at least one copy of the $\mathrm{G}$ allele would benefit more from social support when anticipating a stressor than individuals homozygous for the A allele of rs53576, and that this difference would be indexed by HRV. We also investigated whether this difference would be most apparent during the period of direct social interaction, as suggested by research on the stress-buffering effect of oxytocin on cardiac autonomic control.

\section{Material and Methods}

2.1 Participants Students at the University of Freiburg, Germany were recruited to participate in a study about "behavior in job interviews." Given previous research sex differences in stress-buffering effects of social support (e.g. Kirschbaum, Klauer, Filipp, \& Hellhammer, 1995), only male students and female supporters were included in the study sample. Exclusion criteria included prior participation in a stress induction study, studying psychology, chronic or acute illness, current or previous psychiatric treatment, smoking more than five cigarettes a day, medication use, or substance abuse. Students were only allowed to participate in the study if they were able to bring a close female supporter (e.g., friend, romantic partner, roommate) to the experimental session. Participants were randomly assigned into two groups. The first group (social support condition) brought their social supporter with them, and the second group (no social support condition) came alone. The participants were told to refrain from drug, medication, caffeine and alcohol use for 24 hours before the session. They were instructed to eat as they usually do and then abstain from food two hours prior the session. All participants provided written informed consent and were paid 25 Euros for their participation. Female supporters received a small gift for their participation. The study was approved by the Institutional Review Board of the University of Freiburg.

The sub-sample analysed for the present investigation was stratified on the basis of OXTR genotype. In the large sample $(N=203)$, there were twenty-nine individuals with the AA genotype, nine of whom had to be excluded because of artefacts in the heart rate recording produced by excessive movements. All twenty individuals with the AA genotype with useable heart rate data were included in the current analyses, and thus, a sample of exactly twenty $\mathrm{G}$ allele carriers (GG or GA) was also selected from the large sample. Specifically, $10 \mathrm{GG}$ allele carriers (social support: $\mathrm{n}=5$; no social support: $\mathrm{n}=5$ ) and 10 GA carriers (social support: $\mathrm{n}=5$; no social support: $\mathrm{n}=5$ ) were chosen from the large sample using a random number generator. The sole inclusion criterion for the $\mathrm{G}$ carriers was an evaluable heart rate recording.

\subsection{General Procedure}

All experimental sessions lasted approximately two hours and were scheduled to begin between 4:00 p.m. and 6:30 p.m. to control for diurnal changes in cortisol secretion (Pruessner et al., 1997). The Trier Social Stress Test for Groups (TSST-G) was used for stress induction; this procedure has been 
shown to reliably induce physiological and psychological laboratory stress (von Dawans et al., 2011). Physiological and psychological measures (heart rate, cortisol, subjective stress) were taken throughout the session. Cortisol and subjective stress data have been reported for the large sample (Chen et al., 2011) and are not further considered here.

\subsection{Social Support Manipulation during Preparation Period}

Participants arrived at the laboratory in groups of four to six. Half of the participants brought a female supporter. Immediately after arriving at the laboratory, the female supporters were individually brought to separate rooms. Instructions provided to the social support providers were based directly on those used in Heinrichs et al. (2003). In brief, support providers were instructed to try to be as helpful as possible during the preparation for the mock job interview, and that the specific behaviors that they should engage in were up to them, as we assumed they would know best what would be most helpful to their companion. The participants themselves were led to another room ("waiting room"), where they were instructed that for the purposes of maintaining anonymity and a standardized protocol they should refrain from speaking to each other.

Before the participants were introduced to the experiment, a heart rate recording device was applied. Subsequently, participants were asked to complete validated German versions of the Interpersonal Reactivity Index (IRI) (Davis, 1983), the trait section of the State-Trait-Anxiety Inventory (STAI-T) (Spielberger, et al., 1983), a verbal intelligence test (WST) (Schmidt and Metzler, 1992), the Social Interaction Anxiety Scale (SIAS) (Mattick and Clarke, 1998), and the opennessscale of the Freiburg Personality Inventory (FPI-R) (Fahrenberg et al., 2001). Additionally, data on age, smoking, and trauma, variables known to have an impact on HRV (Task Force of the European Society of Cardiology and the North American Society of Pacing and Electrophysiology, 1996), were collected. Furthermore, subjects were asked about their ethnic origin, as ethnicity has been shown to impact HRV (Task Force of the European Society of Cardiology and the North American Society of Pacing and Electrophysiology, 1996) and also to interact with rs53576 in shaping social behaviors such as emotional support seeking (Kim et al., 2010; Sasaki, Kim, \& Xu, 2011).

Immediately after these baseline measures were taken, participants in the no-support condition started their ten-minute preparation period remaining in the waiting room. In contrast, the participants in the support condition were brought to the separate rooms and completed the preparation phase with their social supporters. Social support was provided exclusively during the preparation period.

\subsection{Stress Manipulation and Recovery}

Participants in both conditions (social support and no social support condition) were then led to another room in which the stress induction protocol was conducted according to the original protocol 
TSST-G (von Dawans et al., 2011). The TSST-G procedure consists of 20-minute acute stress period (including a 12-minute mock job interview period, followed by an 8-minute unanticipated mental arithmetic task period). During the stress manipulation, the participants were separated by dividing walls that prevented visual contact between them. After delivering their speech, the participants were asked to complete a challenging mental arithmetic task. To maintain participants' stress levels, the participants were informed that they could be called upon to answer questions in random order and might be called upon again at any time. Following the stress manipulation, the participants were led back to the waiting room, where they rested for 60 minutes. The heart rate monitors were removed after 25 minutes. At the end of the study, participants were debriefed and compensated.

\subsection{Stress Response Measure}

Heart rate (beat-to-beat intervals) was recorded with a Polar S810i system via the corresponding chest belt (Polar Electro OY, Kempele, Finland) throughout the procedure. We restricted our analyses to the data collected during (1) the preparation phase (excluding the initial four and a half minute of preparation time, which contained significant artifacts resulting from the movement of the participants between rooms), (2) the mock job interview portion of the acute stress exposure episode, and (3) a 90second sitting control interval twenty minutes after the end of the acute stress period. The post-stress sitting control was used as a covariate to control for pre-existing individual differences in HRV. We chose post-stress data for this purpose since HRV measures recorded before the TSST-G could have been biased by participants' anticipatory fears.

Data were then transferred to the Polar Precision Performance Software (Polar Electro OY, Kempele, Finland) and exported as raw beat-to-beat data for further analysis. The raw beat-to-beat data for determination of HRV was processed according to the guidelines of the Task Force of the European Society of Cardiology and the North American Society of Pacing and Electrophysiology (1996) using the Kubios HRV package (Tarvainen et al., 2009). In a first step, the data were visually inspected for abnormal or biologically implausible beats and adjusted or directly excluded. In a second step, the root mean square of successive differences (RMSSD) was calculated for each period from 90 seconds of continuous data during preparation time, during the acute stressor episode and during one 90-second interval twenty minutes after the acute stressor (sitting control). RMSSD was used to operationalize HRV as it is an approved short-term measure of HRV reflecting vagal cardiac influence and has been shown to be less affected by breathing patterns and therefore more robust than other HRV indices (Task Force of the European Society of Cardiology and the North American Society of Pacing and Electrophysiology, 1996).

\subsection{Genotyping}


Using the Masterpure Isolation kit (Epicentre, Madison, United States), buccal epithelial cells were collected in mouthwash samples and DNA was isolated following a standard salting out method. The OXTR rs53576 SNP was analyzed by 5'-nuclease assay. Primers and probes were from Applied Biosystems (TaqManSNO Genotyping Assay). PCR was conducted with a CFX96 fluorescence reading module.

\subsection{Data Analysis}

For analyzing HRV, a three-way analysis of variance (ANOVA) with repeated measures was used [condition (two conditions: social support vs. no social support) by genotype (GG/AG vs. AA) x time (repeated factor: four for HRV during preparation time, eight for HRV during the mock job interview period of the TSST)]. The recessive A allele model (AA vs. G carriers) was used in all analyses, consistent with the genetic analyses carried out in Chen et al., 2011, in which no difference was observed in the GG and GA groups on the outcomes of interest. Whenever the Mauchly test of sphericity indicated heterogeneity of covariance, repeated-measures results were verified with Greenhouse-Geisser corrections. Including the order in which participants were called upon to deliver their speeches as a covariate did not change the reported results; this variable was therefore dropped from the analysis. The areas under the individual response curves with respect to the ground $\left(\mathrm{AUC}_{\mathrm{G}}\right)$ of HRV were calculated with the trapezoid formula, aggregating the four 90 -second intervals during preparation time and the eight 90-second intervals during acute stress (Pruessner et al., 2003). All analyses were conducted using SPSS18 (SPSS). All statistical tests were conducted with $\alpha<.05$.

\section{Results}

\subsection{Participant Characteristics}

Table 1 and Table 2 show characteristics of study groups in GG, AG, and AA genotypes separately. GG, AG, and AA carriers did not differ significantly in demographic and clinical characteristics (Table 1), including self-reported measures of trait anxiety, social anxiety, and social desirability. Individual characteristics that could influence HRV (smoking, trauma, and ethnicity) also did not differ among GG, AG and AA carriers.

\subsection{HRV Response to Stress}

As expected, the psychosocial stress protocol induced stress, indexed by a significant decrease of HRV [main effect of time: $F(4.30,154.50)=25.20, p<.01 ; \eta^{2}=0.41$ ] in all participants.

During the preparation period, the interaction between genotype (AA versus G carriers) and social support on HRV responses was significant when introducing the HRV level recorded during the sitting control as a covariate $\left[F(1,35)=5.14, p=.03, \eta^{2}=0.13\right]$. This result indicates differential response patterns between the genotype groups depending on the presence or absence of social 
support. As predicted, individuals with one or two copies of the $\mathrm{G}$ allele of rs53576 displayed higher heart rate variability values during direct social interaction compared to individuals with the same genotype receiving no support (see Figure 1).

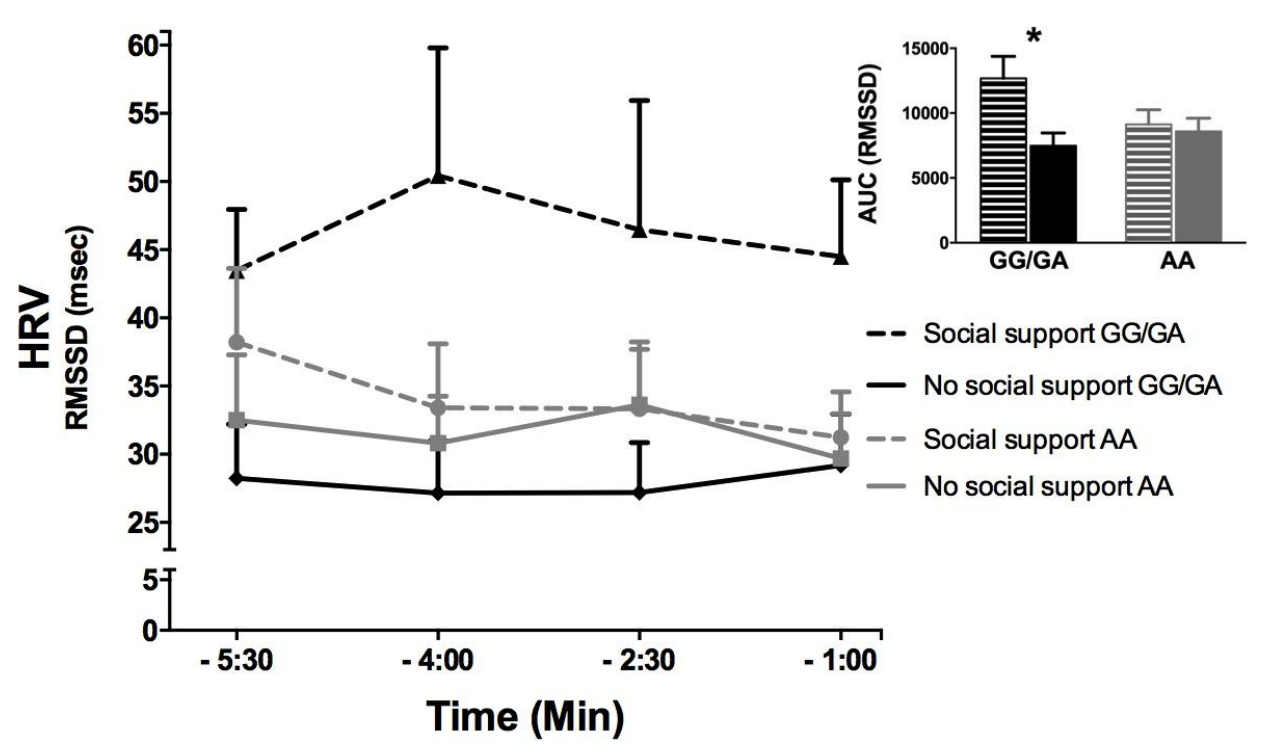

Figure 1 Mean HRV level of 90-second time segments during direct social interaction. Time specifications on the x-axis are relative to the start of the stress period. Error bars represent SEM. Insert: Area under the response curves (AUC) representing aggregated HRV levels through the four measurement points (90-second time segments). For an overview of the AUC means and SEMs see Table 2.

Pre-planned analyses confirmed that in $\mathrm{G}$ allele carriers the attenuation of HRV as a reaction to stress anticipation was significantly smaller in the social support condition compared to the nosupport condition $\left[F(1,17)=8.20, p=.01, \eta^{2}=0.33\right]$, whereas there were no significant differences in HRV response between the social support and no-support condition in individuals homozygous for the A allele $\left[F(1,17)=0.01, p=.91, \eta^{2}=.001\right]$. In sum, social support was associated with higher HRV only in $\mathrm{G}$ allele carriers. In contrast to HRV, heart rate beat-to-beat data were not associated with the OXTR-social support interaction during preparation $\left(F(1,36)=0.59, \mathrm{p}=.45, \eta^{2}=.016\right)$, suggesting that the effects are likely due to parasympathetic influences rather than sympathetic influences.

The interaction between genotype (AA vs. G carriers) and social support on HRV during the acute stress period was marginally significant when introducing the HRV level recorded during the sitting control as a covariate $\left[\mathrm{F}(1,35)=2.83, \mathrm{p}=.10, \eta^{2}=.08\right]$. However, pre-planned analyses within genotype groups revealed similar patterns as those observed in the stress anticipation period. Specifically, the difference in HRV response as a function of social support was marginally significant in $\mathrm{G}$ allele carriers $\left[F(1,17)=4.27, p=.05, \eta^{2}=.20\right]$, whereas again individuals with the AA genotype did not differ in HRV reactivity as a function of social support at all $[F(1,17)=0.004, p$ $\left.=.95, \eta^{2}<.001\right]($ see Figure 2). 


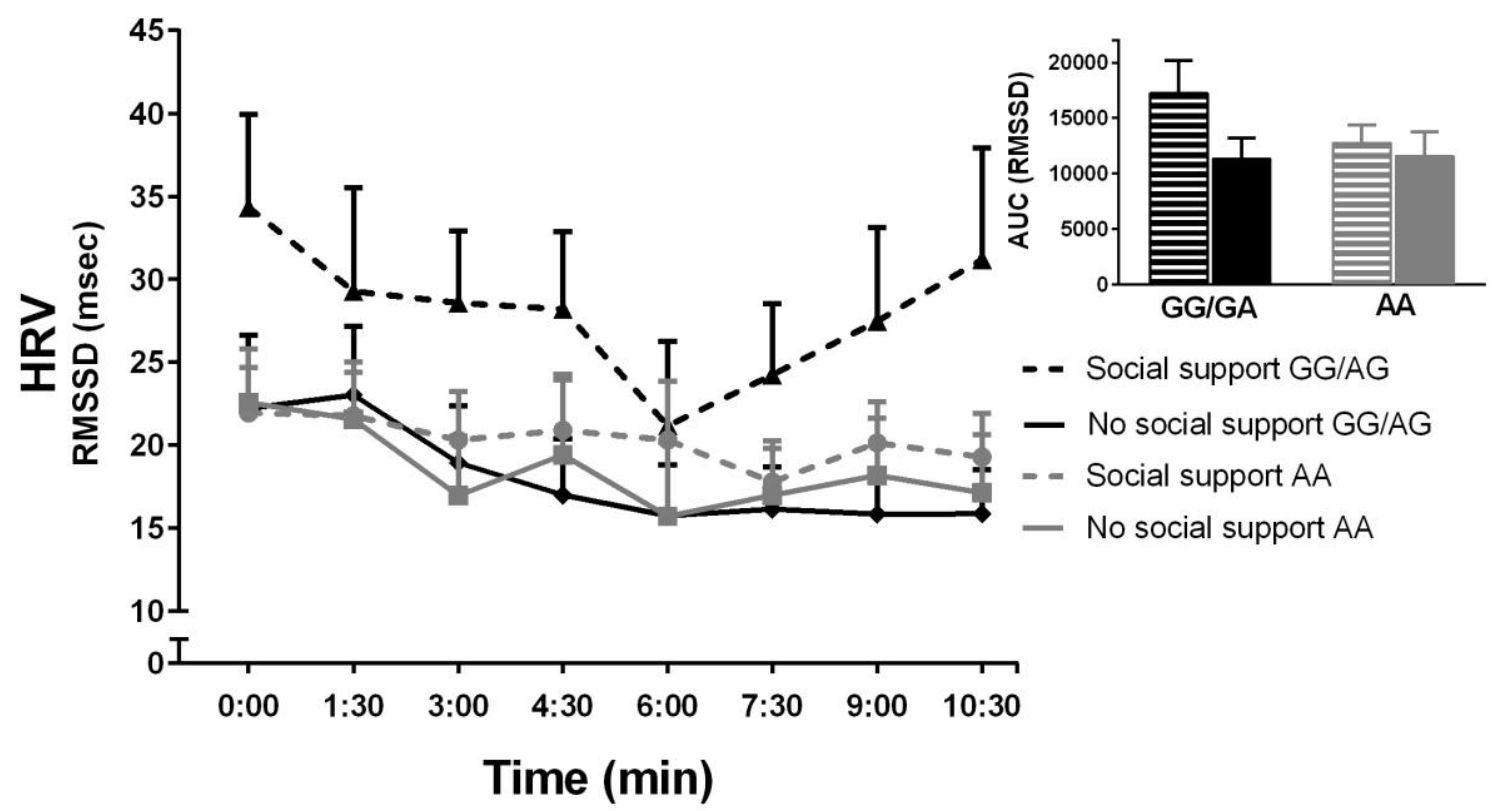

Figure 2 Mean HRV level of 90-second time segments during the mock job interview period of the TSST. Time specifications on the $\mathrm{x}$-axis are relative to the start of the stress period. Error bars represent SEM. Insert: Area under the response curves (AUC) representing aggregated HRV levels through the eight measurement points (90-second time segments). For an overview of the AUC means and SEMs see Table 2.

\section{Discussion}

The results of the present study support previous findings that genetic variation of the oxytocin receptor are associated with alterations in the stress buffering effects of social support. The fact that only individuals with at least one G allele displayed significantly higher HRV during direct social interaction support compared to individuals of the same genotype receiving no social support confirms that the stress-attenuating effects of social support are modulated by genotype.

These data are in line with previous research showing that the oxytocin system plays a key role in regulating social behaviors via the modulation of vagal efferent activity (Porges, 2001) as well as in mediating the buffering effects of social support on physiological reactivity to acute stress (e.g. Heinrichs et al., 2009; Meyer-Lindenberg et al., 2011; Porges, 2001). In addition, the present data are consistent with previous findings of an association between social support and elevated HRV (Maunder et al., 2012), as well as animal studies in which it has been found that oxytocin is associated with stress buffering effects on cardiac autonomic responses via increased vagal tone (Higa et al., 2002). Furthermore, these results confirm research on the $O X T R$ polymorphism rs53576, which have shown that the A allele of rs53576 is associated with deficits in socioemotional domains (BakermansKranenburg and van Ijzendoorn, 2008), reduced empathic accuracy (Rodrigues et al., 2009), lower positive affect (Lucht et al., 2009), heightened physiological stress reactivity (Rodrigues et al., 2009) 
as well as reduced seeking social support seeking during times of distress when it is culturally normative (Kim et al., 2010), and reduced efficacy of social support (Chen et al., 2011).

The combined stress buffering effect of social support and OXTR polymorphism replicates and extends our previous work in which it was found that the combination of social support and the presence of at least one $\mathrm{G}$ allele was associated with reduced cortisol responses (Chen et al., 2011). Whereas our previous study showed reduced cortisol responses over a time period that included the stress period and its recovery, the present results suggest that the stress buffering effects occurred in anticipation of the stressful event as well as during the stressful event. This is in part due to difference in the time course of endocrine and autonomic responses such that endocrine responses occur on the order of magnitude of minutes whereas autonomic responses, particularly cardiac vagal modulation, occur within milliseconds (Looser et al., 2010). Moreover, given that the stress buffering was found even before the stressor actually occurred suggests that the effect is centrally mediated and not a reflexive response to a peripheral pressor stimulus. Thus the present data help to explicate the precise timing and physiological origin of these effects.

Numerous lines of research converge to help clarify the central origins of the stress buffering effects of oxytocin. For example, human studies have suggested alterations in the top-down regulation of central nervous system stress regulation in response to social support via modulation of hypothalamic-limbic functioning in individuals homozygous for the A allele (Tost et al., 2010). Furthermore, both human and animal studies suggest that the amygdala plays a decisive role in the central nervous system stress attenuating effects of OXT (Landgraf and Neumann, 2004; Kirsch et al., 2005; Viviani et al., 2011).

Further elaboration of the exact CNS pathways via which oxytocin produces its stress buffering effects comes from animal studies. Oxytocin-type neurons from the PVN have been shown to synapse on cardiovagal neurons in the NTS, the DMX, and the NA (Luiten et al., 1985; Higa et al., 2002; Coote, 2013). Excitation of these neurons increase vagal outflow and decrease heart rate while having no effect on sympathetic outflow (Higa et al., 2002). Of particular relevance to the present study, these neurons seem to be importantly involved in maintaining greater vagal tone and lower heart rate especially during stressful events (Higa et al., 2002; Higa-Taniguchi et al., 2009). Thus, the present findings that the stress buffering effects of social support on HRV were greater in those carrying the $\mathrm{G}$ allele comport well with this pathway.

Although the present findings that only $\mathrm{G}$ allele carriers display attenuated stress reactivity as a consequence of social support are in line with previous findings, it should be noted that other studies have suggested the opposite pattern. A recently published study by Norman et al. (2012) showed that individuals homozygous for the $\mathrm{G}$ allele displayed a significantly higher sympathetic cardiac reactivity but exhibited no significant differences in parasympathetic cardiac control in response to a psychological stressor (mental arithmetic tasks), compared to A allele carriers. Another study documented 
elevated heart rate reactivity to infant crying in women with two $G$ alleles (Riem et al., 2011). Given that age and gender have a strong impact on both HRV (Task Force of the European Society of Cardiology and the North American Society of Pacing and Electrophysiology, 1996; Berntson et al., 1997) and the effects of social support on stress reactivity (Shumaker and Hill, 1991; Kirschbaum et al., 1995), the differences in the ages of the samples and the gender ratio may explain the divergent findings. Another potentially influential difference is the different measures of physiological stress reactivity used.

Future studies with more diverse samples and varying physiological stress reactivity measurements will be necessary to establish the generalizability of the reported findings. In particular, future research will be necessary to confirm whether the results that we obtained with our all-male sample of participants and all-female sample of social supporters, would generalize when the participants or supporters are of a different sex. As with all genetic association studies, our findings should be replicated in an independent sample and with a larger sample size to establish the robustness of the effects we observed. Another potentially fruitful direction for future research would be to investigate whether the type of relationship between supporter and support provider (e.g., romantic versus nonromantic), as well as type of support provided (e.g. with or without physical contact), influences stress-buffering effects of social support mediated by the oxytocin system. It also remains to be seen whether individuals with the AA genotype may benefit more strongly from implicit or other forms of social support than those offered by the supporters in this study. More broadly, our results suggest that including measures of genetic variability in future studies on social support may help to reconcile discrepant findings (which may, for example, turn out to be influenced by dissimilar distributions of genetic variants present in different study samples) and help overall to refine our understanding of the effects of social support on stress.

In sum, the present study has documented the potential role of genetic variability in individual differences in the effectiveness of social support. The present data suggest that social relations directly impact human stress-regulation mechanisms. These fundamental internal processes have important and long-lasting consequences for health and well-being, highlighting the value of continued research on the mechanisms by which social support influences stress regulation, what individual differences modulate the effectiveness of social support, and what factors influence how long the positive effects of social support persist. 


\section{Acknowledgments}

We thank Dr. Bernadette von Dawans for assistance during study preparation, and Eva Bareth,

Daniela Conrad, Melanie Filser, Angela Herwede, Irini Johann, Juliane Kant, Theresa Lueer, Maurice Mink, Maxi Pannicke, Marina Pohl, Anna Sartori, Katja Schlichtig, Alexandra Schultz, Philomena Storz, and Julia-Caroline Walther for assistance during data collection. This study was supported by Swiss National Science Foundation Research Grant SNSF PP001-114788 (to M.H.). M.H., F.S.C, and R.K. gratefully acknowledge grant support from the Deutsche Forschungsgemeinschaft (HE 5310/11). F.S.C. gratefully acknowledges support from a research fellowship of the AXA Foundation.

L.K.H. was supported by a National Institute of Aging grant (5T32AG000029-38). 


\section{References}

Bakermans-Kranenburg, M.J., van Ijzendoorn, M.H., 2008. Oxytocin receptor (OXTR) and serotonin transporter (5-HTT) genes associated with observed parenting. Social Cognitive and Affective Neuroscience, 3, 128-134. http://dx.doi.org/10.1093/scan/nsn004

Berntson, G.G., Thomas Bigger, J., Eckberg, D.L., Grossman, P., Kaufmann, P.G., Malik, M., et al., 1997. Heart rate variability: Origins, methods, and interpretive caveats. Psychophysiology, 34, 623-648. http://dx.doi.org/10.1111/j.1469-8986.1997.tb02140.x

Carter, C.S. (1998). Neuroendocrine perspectives on social attachment and love. Psychoneuroendocrinology, 23(8), 779-818. http://dx.doi.org/10.1016/S0306-4530(98)000559

Chen, F.S., Kumsta, R., von Dawans, B., Monakhov, M., Ebstein, R.P., Heinrichs, M., 2011. Common oxytocin receptor gene (OXTR) polymorphism and social support interact to reduce stress in humans. Proceedings of the National Academy of Sciences, 108, 19937-19942. http://dx.doi.org/10.1073/pnas.1113079108

Cohen, S., 1988. Psychosocial models of the role of social support in the etiology of physical disease. Health Psychology, 7, 269-297. http://dx.doi.org/10.1037/0278-6133.7.3.269

Coote, J.H., 2013. Myths and realities of the cardiac vagus. The Journal of Physiology, 591(17), 40734085. http://dx.doi.org/10.1113/jphysiol.2013.257758

Davis, M.H., 1983. Measuring individual differences in empathy: Evidence for a multidimensional approach. Journal of Personality and Social Psychology, 44, 113-126. http://dx.doi.org/10.1177/0146167203029006008

Ditzen, B., Schmidt, S., Strauss, B., Nater, U.M., Ehlert, U., Heinrichs, M., 2008. Adult attachment and social support interact to reduce psychological but not cortisol responses to stress. Journal of Psychosomatic Research, 64, 479-486.http://dx.doi.org/10.1016/j.jpsychores.2007.11.011

Fahrenberg, J., Hampel, R., Selg, H., 2001. FPI-R: Das Freiburger Persönlichkeitsinventar (7th Edition). Göttingen: Hogrefe.

Friedman, B. H., \& Thayer, J. F. (1998). Autonomic balance revisited: panic anxiety and heart rate variability. Journal of Psychosomatic Research, 44(1), 133-151.

Gianaros, P. J., Van der Veen, F. M., \& Jennings, J. R. (2004). Regional cerebral blood flow correlates with heart period and high-frequency heart period variability during workingmemory tasks: Implications for the cortical and subcortical regulation of cardiac autonomic activity. Psychophysiology, 41(4), 521-530._http://dx.doi.org/10.1111/14698986.2004.00179.x

Gimpl, G., Fahrenholz, F., 2001. The oxytocin receptor system: structure, function, and regulation. Physiological Reviews, 81, 629-683.

Heinrichs, M., Baumgartner, T., Kirschbaum, C., Ehlert, U., 2003. Social support and oxytocin 
interact to suppress cortisol and subjective responses to psychosocial stress. Biological Psychiatry, 54, 1389-1398. http://dx.doi.org/10.1016/S0006-3223(03)00465-7

Heinrichs, M., von Dawans, B., Domes, G., 2009. Oxytocin, vasopressin, and human social behavior. Frontiers in Neuroendocrinology, 30, 548-557. http://dx.doi.org/10.1016/j.yfrne.2009.05.005

Higa-Taniguchi, K.T., Felix, J.V., Michelini, L.C., 2009. Brainstem oxytocinergic modulation of heart rate control in rats: effects of hypertension and exercise training. Experimental Physiology, 94(11), 1103-1113. http://dx.doi.org/10.1113/expphysiol.2009.049262

Higa, K.T., Mori, E., Viana, F.F., Morris, M., Michelini, L.C., 2002. Baroreflex control of heart rate by oxytocin in the solitary-vagal complex. American Journal of Physiology-Regulatory, Integrative and Comparative Physiology, 282(2), R537-R545. http://dx.doi.org/10.1152/ajpregu.00806.2000

Inoue, T., Kimura, T., Azuma, C., Inazawa, J., Takemura, M., Kikuchi, T., et al., 1994. Structural organization of the human oxytocin receptor gene. Journal of Biological Chemistry, 269, 32451-32456.

Kim, H.S., Sherman, D.K., Taylor, S.E., 2008. Culture and social support. American Psychologist, 63, 518-526. http://dx.doi.org/10.1037

Kim, H.S., Sherman, D.K., Sasaki, J.Y., Xu, J., Chu, T.Q., Ryu, C., . . Taylor, S.E. (2010). Culture, distress, and oxytocin receptor polymorphism (OXTR) interact to influence emotional support seeking. Proceedings of the National Academy of Sciences, 107(36), 15717-15721. http://dx.doi.org/10.1073/pnas.1010830107

Kirsch, P., Esslinger, C., Chen, Q., Mier, D., Lis, S., Siddhanti, S., et al., 2005. Oxytocin modulates neural circuitry for social cognition and fear in humans. The Journal of Neuroscience, 25, 11489-11493. http://dx.doi.org/10.1523/JNEUROSCI.3984-05.2005

Kirschbaum, C., Klauer, T., Filipp, S.H., Hellhammer, D.H., 1995. Sex-specific effects of social support on cortisol and subjective responses to acute psychological stress. Psychosomatic Medicine, 57, 23-31.

Kok, B. E., \& Fredrickson, B. L. (2010). Upward spirals of the heart: Autonomic flexibility, as indexed by vagal tone, reciprocally and prospectively predicts positive emotions and social connectedness. Biological Psychology, 85(3), 432-436. http://dx.doi.org/ 10.1016/j.biopsycho. 2010.09.005

Kumsta, R., Heinrichs, M., 2013. Oxytocin, stress and social behavior: neurogenetics of the human oxytocin system. Current Opinion in Neurobiology, 23, 11-16. http://dx.doi.org/10.1016/j.conb.2012.09.004

Landgraf, R., Neumann, I.D., 2004. Vasopressin and oxytocin release within the brain: a dynamic concept of multiple and variable modes of neuropeptide communication. Frontiers in Neuroendocrinology, 25, 150-176. http://dx.doi.org/10.1016/j.yfrne.2004.05.001 
Lepore, S.J., Allen, K.A., Evans, G.W., 1993. Social support lowers cardiovascular reactivity to an acute stressor. Psychosomatic Medicine, 55, 518-524.

Looser, R.R., Metzenthin, P., Helfricht, S., Kudielka, B.M., Loerbroks, A., Thayer, J.F., Fischer, J.E., 2010. Cortisol is significantly correlated with cardiovascular responses during high levels of stress in critical care personnel. Psychosomatic Medicine, 72(3), 281-289. http://dx.doi.org/10.1097/PSY.0b013e3181d35065

Lucht, M.J., Barnow, S., Sonnenfeld, C., Rosenberger, A., Grabe, H.J., Schroeder, W., et al., 2009. Associations between the oxytocin receptor gene (OXTR) and affect, loneliness and intelligence in normal subjects. Progress in Neuro-Psychopharmacology and Biological Psychiatry, 33, 860-866. http://dx.doi.org/10.1016/j.pnpbp.2009.04.004

Luiten, P., Ter Horst, G., Karst, H., Steffens, A., 1985. The course of paraventricular hypothalamic efferents to autonomic structures in medulla and spinal cord. Brain Research, 329(1), 374378. http://dx.doi.org/10.1016/0006-8993(85)90554-2

Mattick, R.P., Clarke, J.C., 1998. Development and validation of measures of social phobias scrutiny fear and social interaction anxiety. Behaviour Research and Therapy, 36, 455-470. http://dx.doi.org/10.1016/S0005-7967(97)10031-6

Maunder, R., Nolan, R., Hunter, J., Lancee, W., Steinhart, A., \& Greenberg, G. (2012). Relationship between social support and autonomic function during a stress protocol in ulcerative colitis patients in remission. Inflammatory Bowel Diseases, 18(4), 737-742. http://dx.doi.org/10.1002/ibd.21794

Meyer-Lindenberg, A., Domes, G., Kirsch, P., Heinrichs, M., 2011. Oxytocin and vasopressin in the human brain: social neuropeptides for translational medicine. Nature Reviews Neuroscience, 12, 524-538. http://dx.doi.org/10.1038/nrn3044

Norman, G.J., Hawkley, L., Luhmann, M., Ball, A.B., Cole, S.W., Berntson, G.G., et al., 2012. Variation in the oxytocin receptor gene influences neurocardiac reactivity to social stress and HPA function: A population based study. Hormones and Behavior, 61, 134-139. http://dx.doi.org/10.1016/j.yhbeh.2011.11.006

Porges, S. W. (1997). Emotion: An Evolutionary By-Product of the Neural Regulation of the Autonomic Nervous System. Annals of the New York Academy of Sciences, 807(1), 62-77. http://dx.doi.org/10.1111/j.1749-6632.1997.tb51913.x

Porges, S. W. (2001). The polyvagal theory: phylogenetic substrates of a social nervous system. International Journal of Psychophysiology, 42(2), 123-146. http://dx.doi.org/10.1016/S01678760(01)00162-3

Pruessner, J.C., Wolf, O.T., Hellhammer, D.H., Buske-Kirschbaum, A., von Auer, K., Jobst, S., et al., 1997. Free Cortisol Levels after awakening: A reliable biological marker for the assessment of adrenocortical activity. Life Sciences, 61, 2539-2549. http://dx.doi.org/10.1016/S0024- 
3205(97)01008-4

Pruessner, J.C., Kirschbaum, C., Meinlschmid, G., Hellhammer, D.H., 2003. Two formulas for computation of the area under the curve represent measures of total hormone concentration versus time-dependent change. Psychoneuroendocrinology, 28, 916-931. http://dx.doi.org/10.1016/S0306-4530(02)00108-7

Riem, M.M.E., Pieper, S., Out, D., Bakermans-Kranenburg, M.J., van Ijzendoorn, M.H., 2011. Oxytocin receptor gene and depressive symptoms associated with physiological reactivity to infant crying. Social Cognitive and Affective Neuroscience 6, 294-300. http://dx.doi.org/10.1093/scan/nsq035

Rodrigues, S.M., Saslow, L.R., Garcia, N., John, O.P., Keltner, D., 2009. Oxytocin receptor genetic variation relates to empathy and stress reactivity in humans. Proceedings of the National Academy of Sciences, 106, 21437-21441. http://dx.doi.org/10.1073/pnas.0909579106

Rogers, R.C., Hermann, G.E., 1986. Hypothalamic paraventricular nucleus stimulation-induced gastric acid secretion and bradycardia suppressed by oxytocin antagonist. Peptides, 7(4), 695700. http://dx.doi.org/10.1016/0196-9781(86)90046-X

Saphire-Bernstein, S., Way, B.M., Kim, H.S., Sherman, D.K., Taylor, S.E., 2011. Oxytocin receptor gene (OXTR) is related to psychological resources. Proceedings of the National Acadademy of Science, 108, 15118-15122. http://dx.doi.org/10.1073/pnas.1113137108

Sasaki, J., Kim, H., \& Xu, J. (2011). Religion and well-being: An analysis of an oxytocin receptor polymorphism (OXTR) and culture. Journal of Cross-Cultural Psychology, 42, 1394-1405.

Schmidt, K.H., Metzler, P., 1992. Wortschatztest WST [Vocabulary Test]. Beltz, Weinheim.

Shumaker, S.A., Hill, D.R., 1991. Gender differences in social support and physical health. Health Psychology, 10, 102-111. http://dx.doi.org/10.1037/0278-6133.10.2.102

Smith, T. W., Cribbet, M. R., Nealey-Moore, J. B., Uchino, B. N., Williams, P. G., MacKenzie, J., \& Thayer, J. F. (2011). Matters of the variable heart: respiratory sinus arrhythmia response to marital interaction and associations with marital quality. Journal of Personality and Social Psychology, 100(1), 103. http://dx.doi.org/10.1037/a0021136

Spielberger, C.D., Gorssuch, R.L., Lushene, P.R., Vagg, P.R., Jacobs, G.A., 1983. Manual for the State-Trait Anxiety Inventory. Consulting Psychologists Press, Inc.

Tarvainen, M.P., Niskanen, J.P., Lipponen, J.A., Ranta-Aho, P.O., Karjalainen, P.A., 2009. Kubios HRV-a software for advanced heart rate variability analysis. In: 4th European Conference of the International Federation for Medical and Biological Engineering. Springer, Berlin, pp. 1022-1025. http://dx.doi.org/10.1007/978-3-540-89208-3 243

Task Force of the European Society of Cardiology and the North American Society of Pacing Electrophysiology, 1996. Heart rate variability: standards of measurement, physiological 
interpretation, and clinical use. Circulation 93, 1043-1065. http://dx.doi.org/10.1161/

01.CIR.93.5.1043

Taylor, S.E., Welch, W.T., Kim, H.S., Sherman, D.K., 2007. Cultural differences in the impact of social support on psychological and biological stress responses. Psychological Science, 18, 831-837. http://dx.doi.org/10.1111/j.1467-9280.2007.01987.x

Thayer, J.F., Ahs, F., Fredrikson, M., Sollers Iii, J.J., Wager, T.D., 2012. A meta-analysis of heart rate variability and neuroimaging studies: Implications for heart rate variability as a marker of stress and health. Neuroscience \& Biobehavioral Reviews, 36, 747-756. http://dx.doi.org/10.1016/j.neubiorev.2011.11.009

Thayer, J.F., Hansen, A.L., Johnsen, B.H., 2008. Non-invasive assessment of autonomic influences on the heart: Impedance cardiographyand heart rate variability. In L. Leuken \& L. C. Gallo (Eds.), Handbook of physiological research methods in health psychology. Thousand Oaks, CA: Sage, pp. 183-209.

Thayer, J.F., Lane, R.D., 2009. Claude Bernard and the heart and brain connection: Further elaboration of a model of neurovisceral integration. Neuroscience \& Biobehavioral Reviews, 33, 81-88. http://dx.doi.org/10.1016/j.neubiorev.2008.08.004

Tost, H., Kolachana, B., Hakimi, S., Lemaitre, H., Verchinski, B.A., Mattay, V.S., et al., 2010. A common allele in the oxytocin receptor gene (OXTR) impacts prosocial temperament and human hypothalamic-limbic structure and function. Proceedings of the National Academy of Sciences, 107, 13936-13941. http://dx.doi.org/10.1073/pnas.1003296107

Uchino, B.N., Cacioppo, J.T., Kiecolt-Glaser, J.K., 1996. The relationship between social support and physiological processes: A review with emphasis on underlying mechanisms and implications for health. Psychological Bulletin, 119, 488-531. http://dx.doi.org/10.1037/00332909.119.3.488

Viviani, D., Charlet, A., van den Burg, E., Robinet, C., Hurni, N., Abatis, M., et al., 2011. Oxytocin selectively gates fear responses through distinct outputs from the central amygdala. Science, 333, 104-107. http://dx.doi.org/10.1126/science.1201043

von Dawans, B., Kirschbaum, C., Heinrichs, M., 2011. The Trier Social Stress Test for Groups (TSST-G): A new research tool for controlled simultaneous social stress exposure in a group format. Psychoneuroendocrinology, 36, 514-522. http://dx.doi.org/10.1016/j.psyneuen.2010.08.004

Weihs, K. L., Simmens, S. J., Mizrahi, J., Enright, T. M., Hunt, M. E., \& Siegel, R. S. (2005). Dependable social relationships predict overall survival in Stages II and III breast carcinoma patients. Journal of Psychosomatic Research, 59(5), 299-306. http://dx.doi.org/10.1016/j.jpsychores.2004.12.002

$\mathrm{Wu}$, S., et al., 2005. Positive association of the oxytocin receptor gene (OXTR) with autism in the 
Chinese Han population. Biological Psychiatry 58, 74-77.

http://dx.doi.org/10.1016/j.biopsych.2005.03.013 
Table 1. Characteristics of study groups

\begin{tabular}{|c|c|c|c|c|}
\hline Characteristics & $\begin{array}{l}\text { GG } \\
(n=10)\end{array}$ & $\begin{array}{l}\mathrm{AG} \\
(n=10)\end{array}$ & $\begin{array}{l}\text { AA } \\
(n=20)\end{array}$ & $\begin{array}{l}\text { Group } \\
\text { Comparison }\end{array}$ \\
\hline Age (y) & $22.6(1.8)$ & $22.9(1.5)$ & $23.5(3.3)$ & $F(2,36)=0.44, \mathrm{~ns}$ \\
\hline Verbal intelligence & $33.9(2.7)$ & $33.3(1.6)$ & $33.6(1.9)$ & $F(2,35)=0.21, \mathrm{~ns}$ \\
\hline Trait anxiety & $32.1(9.1)$ & $35.2(5.9)$ & $35.3(8.5)$ & $F(2,35)=0.62, \mathrm{~ns}$ \\
\hline Social anxiety & $14.6(10.1)$ & $16.9(7.2)$ & $19.1(9.7)$ & $F(2,35)=0.82, \mathrm{~ns}$ \\
\hline Empathy & $53.1(7.0$ & $51.7(8.5)$ & $54.6(7.2)$ & $F(2,35)=0.33, \mathrm{~ns}$ \\
\hline Social desirability & $7.5(1.8)$ & $7.7(2.9)$ & $7.2(2.1)$ & $F(2,35)=0.29, \mathrm{~ns}$ \\
\hline Smoking & $1(10 \%)$ & $2(20 \%)$ & $0(0 \%)$ & $\begin{array}{l}\chi^{2}(2, N=40) \\
=0.1, \mathrm{~ns}\end{array}$ \\
\hline Trauma & $4(40 \%)$ & $6(60 \%$ & $9(45 \%)$ & $\begin{array}{l}\chi^{2}(4, N=39) \\
=0.4, \mathrm{~ns}\end{array}$ \\
\hline Ethnicity European & $10(100 \%)$ & $10(100 \%)$ & $19(95 \%)$ & $\begin{array}{l}\chi^{2}(2, N=40) \\
=1.0, \mathrm{~ns}\end{array}$ \\
\hline
\end{tabular}

Note. Cells values indicate group means. In GG, AG, and AA columns, values in parentheses are SEM (percentages). ns, nonsignificant. 
Table 2. Differences between social support condition and no social support condition in HRV for genotype groups.

\begin{tabular}{llll}
\hline \hline Variable & $\begin{array}{l}\text { GG } \\
(n=10)\end{array}$ & $\begin{array}{l}\text { AG } \\
(n=10)\end{array}$ & $\begin{array}{l}\text { AA } \\
(n=20)\end{array}$ \\
\hline $\begin{array}{l}\text { Aggregated HRV level } \\
\text { AUC): preparation }\end{array}$ & $-5155(1565.5)$ & $-5251(1707.9)$ & $-535(737.9)$ \\
$\begin{array}{l}\text { Aggregated HRV level } \\
\text { (AUC): acute stress }\end{array}$ & $-6995(2893.8)$ & $-4871(2309.4)$ & $-1184(1317.3)$ \\
\hline \hline
\end{tabular}

Note. Values are mean (SEM). Areas under the individual response curves (AUC) aggregate the four HRV levels during direct social interaction (preparation) and the eight HRV levels during the mock job interview period of the TSST (acute stress). Negative values represent a relative decrease in HRV (i.e. a protective effect of social support). 